\title{
Predictive values of urine paraquat concentration, dose of poison, arterial blood lactate and APACHE II score in the prognosis of patients with acute paraquat poisoning
}

\author{
XIAO-WEI LIU ${ }^{1}$, TAO MA ${ }^{1}, \mathrm{LU}_{-} \mathrm{LU} \mathrm{LI}^{1}, \mathrm{BO} \mathrm{QU}^{2}$ and $\mathrm{ZHI} \mathrm{LIU}{ }^{1}$ \\ ${ }^{1}$ Department of Emergency, The First Affiliated Hospital of China Medical University; \\ ${ }^{2}$ Department of Biostatistics, School of Public Health, China Medical University, Shenyang, Liaoning 110001, P.R. China
}

Received May 29, 2015; Accepted February 14, 2017

DOI: $10.3892 /$ etm.2017.4463

\begin{abstract}
The present study investigated the predictive values of urine paraquat (PQ) concentration, dose of poison, arterial blood lactate and Acute Physiology and Chronic Health Evaluation (APACHE) II score in the prognosis of patients with acute PQ poisoning. A total of 194 patients with acute PQ poisoning, hospitalized between April 2012 and January 2014 at the First Affiliated Hospital of P.R. China Medical University (Shenyang, China), were selected and divided into survival and mortality groups. Logistic regression analysis, receiver operator characteristic (ROC) curve analysis and Kaplan-Meier curve were applied to evaluate the values of urine paraquat (PQ) concentration, dose of poison, arterial blood lactate and (APACHE) II score for predicting the prognosis of patients with acute PQ poisoning. Initial urine PQ concentration $\left(\mathrm{C}_{0}\right)$, dose of poison, arterial blood lactate and APACHE II score of patients in the mortality group were significantly higher compared with the survival group (all $\mathrm{P}<0.05)$. Logistic regression analysis revealed that $\mathrm{C}_{0}$, dose of poison and arterial blood lactate correlated with mortality risk of acute $\mathrm{PQ}$ poisoning (all $\mathrm{P}<0.05$ ). ROC curve analysis suggested that the areas under the curve (AUC) values of $\mathrm{C}_{0}$, dose of poison, arterial blood lactate and APACHE II score in predicting the mortality of patients within 28 days were $0.921,0.887,0.808$ and 0.648 , respectively. The $\mathrm{AUC}$ of $\mathrm{C}_{0}$ for predicting early and delayed mortality were 0.890 and 0.764 , respectively. The AUC values of urine paraquat concentration the day after poisoning $\left(\mathrm{C}_{\mathrm{sec}}\right)$ and the rebound rate of urine paraquat concentration in predicting the mortality of patients within 28 days were 0.919 and 0.805 , respectively.
\end{abstract}

Correspondence to: Dr Zhi Liu, Department of Emergency, The First Affiliated Hospital of China Medical University, 155 Nanjing Street, Heping, Shenyang, Liaoning 110001, P.R. China

E-mail: cmulh_lz@126.com

Key words: urine paraquat concentration, arterial blood lactate, Acute Physiology and Chronic Health Evaluation II score, paraquat poisoning, prognosis
The 28-day survival rate of patients with $\mathrm{C}_{0} \leq 32.2 \mu \mathrm{g} / \mathrm{ml}$ $(42 / 71 ; 59.2 \%)$ was significantly higher when compared with patients with $\mathrm{C}_{0}>32.2 \mu \mathrm{g} / \mathrm{ml}(38 / 123 ; 30.9 \%)$. These results suggest that the initial urine PQ concentration may be the optimal index for predicting the prognosis of patients with acute PQ poisoning. Additionally, dose of poison, arterial blood lactate, $\mathrm{C}_{\mathrm{sec}}$ and rebound rate also have referential significance.

\section{Introduction}

Paraquat (PQ; 1,1'-dimethyl-4,4'-bipyridinium), also known as Gramoxone or methyl viologen, is an organic nitrogen heterocyclic herbicide utilized in agriculture worldwide $(1,2)$. PQ can gradually damage human health via bioaccumulation in the food chain, and ingestion of $>15-30 \mathrm{ml}$ of $20 \%$ (w/v) PQ can be fatal in humans (3). Severe PQ poisoning affects multiple organs, predominantly the lung, liver, kidneys, adrenal cortex and myocardium (4). Severe cases of acute PQ poisoning may also exhibit neurological symptoms, such as dysphoria, somnolence, and impaired consciousness (5). It is estimated that $250,000-370,000$ individuals succumb to pesticide poisoning each year globally, and $>90 \%$ of patients with acute poisoning have attempted suicide by ingesting concentrated PQ in a liquid form (6). The principal biochemical mechanism of PQ poisoning is based on redox cycling and intracellular oxidative stress generation, and pulmonary fibrosis and edema are the major clinical symptoms (7). A previous study has investigated various treatment modalities for acute PQ poisoning, but the fatality rate remains high (8). In this context, biomarkers for the prognosis and clinical monitoring of acute PQ poisoning are urgently required to guide appropriate treatment plans and develop future PQ antidotes (2).

To date, several diagnostic methods have been developed to evaluate the severity of acute PQ poisoning in patients, including urine PQ concentration, the Acute Physiology and Chronic Health Evaluation (APACHE) II score, arterial blood lactate and ingestion dose of PQ $(9,10)$. Plasma and urine PQ concentrations obtained within the first $24 \mathrm{~h}$ after ingestion are excellent predictors of the outcomes of PQ poisoning (2). However, the measurement of plasma PQ concentrations requires personnel support and apparatus, including strict 
quality standards and controls to predict severity, which may not be readily available in most hospitals because of the expense of equipment and the associated technical problems (11). Therefore, urine analysis may have a higher predictive value and may more rapidly assess prognosis compared with estimating PQ concentrations in plasma (7). APACHE II is a severity-of-disease classification system applied within $24 \mathrm{~h}$ of admission of a patient to an intensive care unit (ICU), which has been extensively used in the prediction of the outcomes of PQ poisoning (12). Lactate estimation is a prognostic tool that can predict the mortality rate among patients with severe sepsis and ST elevation myocardial infarction $(13,14)$. It is used in numerous critical-care patients, including patients who have recently undergone surgery and those with burns, trauma, and septic shock. Arterial blood lactate is a manifestation of organ dysfunction and has good predictive power in evaluating patient prognosis during acute PQ poisoning $(9,13)$. Ingestion of $>15-30 \mathrm{ml}$ of $20 \%$ (w/v) PQ can lead to mortality from multi-organ failure or respiratory failure within a month of poisoning (3). Due to the fact that PQ intoxication has a dose-response relationship, it is appropriate to evaluate the $\mathrm{PQ}$ dose at a given time (10). In this regard, the present study investigated the values of urine PQ concentration, dose of poison, arterial blood lactate and APACHE II score for predicting the prognosis of acute $\mathrm{PQ}$ poisoning.

\section{Materials and methods}

Subjects. A total of 194 patients with acute PQ poisoning who were hospitalized between April 2012 and January 2014 at the First Affiliated Hospital of China Medical University (Shenyang, China) in the Intensive Care Unit of the Emergency Department were selected as the research subjects. Selection criteria were as follows: i) Patients were selected according to the diagnostic criteria of acute PQ poisoning in The Diagnosis and Treatment of Acute Poisoning (15), and clinical diagnostic results were available; ii) patients were treated within $24 \mathrm{~h}$ after poisoning, and there was evidence of $\mathrm{PQ}$ poisoning or circumstantial evidence of PQ poisoning provided by patients or caregivers; iii) patients had not received therapy before admission to the hospital, including blood purification treatment or gastric lavage; and iv) patients had no history of serious heart, liver, kidney and lung diseases. Exclusion criteria were: i) Patients had a history of chronic kidney disease; ii) PQ combined with other drug poisoning; iii) emergency observation was no more than $24 \mathrm{~h}$; iv) patients were admitted $24 \mathrm{~h}$ after they took poison; and v) pregnant women, patients that gave up therapy or died due to reasons unrelated to PQ poisoning. A total of 194 patients ( $72 \mathrm{men} ; 122$ women) aged 12-75 years (mean, $32.51 \pm 12.72$ years) were enrolled in this study. The characteristics of PQ pesticide treatment were as follows: $20 \% \mathrm{PQ}$ pesticide dose, $10-150 \mathrm{ml}$; mean dose, $55.00 \pm 33.27 \mathrm{ml}$; time interval between PQ exposure and the first urine sample, $0.5-11.5 \mathrm{~h}$; and mean time interval, $4.8 \pm 6.9 \mathrm{~h}$.

This study was performed in accordance with the standard in medical ethics and was approved by the Medical Ethics committee the First Affiliated Hospital of China Medical University (Shenyang, China). Written informed consent was obtained prior to treatment from patients or their family members. This study conformed to the guidelines outlined in the Declaration of Helsinki (16).

Data collection. Patients received auxiliary examinations immediately, and $4 \mathrm{~h}$ (immediately following the peak of drug plasma concentration) and within $24 \mathrm{~h}$ after poisoning, peripheral venous blood and arterial blood samples were collected and immediately sent to the Pathology Department to test the relevant indices. Patient data were collected by two clinicians based on the unified form, including sex and age of patients, poison dose, the time interval between PQ exposure and the first urine sample, results of routine blood and urine tests, fasting blood glucose, liver function, kidney function, myocardial enzymes, serum potassium, artery blood gas analysis, and amylase detection at admission to hospital and at deterioration or improvement. Within the first $24 \mathrm{~h}$ after admission to hospital, the APACHE II score was evaluated according to the general condition and vital signs of patients and the worst inspection results. Urine PQ concentration of patients was dynamically monitored. Initial urine PQ concentration $\left(\mathrm{C}_{0}\right)$ and urine PQ concentration after hemoperfusion (HP) were calculated. $\mathrm{C}_{\mathrm{x}}$ indicates the urine $\mathrm{PQ}$ concentration after undergoing $\mathrm{HP} x$ times, and $\mathrm{C}_{\mathrm{sec}}$ indicates the urine $\mathrm{PQ}$ concentration the following day after HP was measured. The rebound rate of urine PQ concentration the following day after HP was also calculated, as follows: Rebound rate $=\left(\mathrm{C}_{\mathrm{sec}}-\mathrm{C}_{\mathrm{la}}\right) /_{\mathrm{la}}$, where $\mathrm{C}_{\mathrm{la}}$ is urine $\mathrm{PQ}$ concentration after the final round of HP. Urine PQ concentration was detected each day during the observation period until the PQ concentration in urine was below the detectable level for 2 consecutive days, which was defined as the point at which the urinary concentration became negative.

Patients were divided into either the mortality group, which included an early mortality subgroup (fatality within 7 days after poisoning) and a delayed mortality subgroup (fatality between 7-28 days after poisoning), or the survival group (alive at the 28-day follow-up). There were 80 patients in the survival group ( 29 men and 51 women; mean age, $30.58 \pm 12.34$ years) and 114 patients in the mortality group (43 men and 71 women; mean age, $33.68 \pm 12.19$ years). The levels of all the indices between the mortality and survival groups were compared, the comparable indices were analyzed, and superior predictors of mortality in patients were explored using receiver operator characteristic (ROC) curve.

Treatment methods. The 194 patients were treated with unified treatment options, including: i) removing unabsorbed poison via gastric lavage and catharsis (oral administration of $100-250 \mathrm{ml}$ of $20 \%$ mannitol); ii) excluding absorbed poison via $\mathrm{HP}$, hydration and diuresis; iii) antioxidant therapy in the form of applying vitamins $\mathrm{C}$ and $\mathrm{E}$; and iv) symptomatic and supportive treatments, including organ function support and correction of acid-base and electrolyte imbalance.

Detection of urine paraquat concentration. The PQ concentration in urine was detected using colorimetric methods based on sodium hyposulfite (17). Under alkaline conditions, the sodium hyposulfite deoxidizes PQ into blue-colored products. Based on the change in color at different concentrations of PQ dichloride and excess sodium hyposulfite under alkaline conditions, a standard curve for determining 
Table I. Comparisons of all indexes between survival and mortality groups.

\begin{tabular}{|c|c|c|c|c|}
\hline Characteristic & $\begin{array}{l}\text { Survival group } \\
\qquad(\mathrm{n}=80)\end{array}$ & $\begin{array}{l}\text { Mortality group } \\
\qquad(\mathrm{n}=114)\end{array}$ & $t / \chi^{2}$ & P-value \\
\hline Age, years & $30.58 \pm 12.34$ & $33.68 \pm 12.19$ & 1.530 & 0.128 \\
\hline Sex, N (\%) & & & 1.511 & 0.223 \\
\hline Male & $29(32.58)$ & $43(40.09)$ & & \\
\hline Female & $51(67.42)$ & $71(59.91)$ & & \\
\hline Dose of poison, $\mathrm{ml}$ & $30.72 \pm 15.14$ & $72.52 \pm 31.42$ & 12.31 & $<0.001^{\mathrm{a}}$ \\
\hline Mean arterial pressure, $\mathrm{mmHg}$ & $92.29 \pm 17.08$ & $88.67 \pm 15.94$ & 1.512 & 0.132 \\
\hline Heart rate, beats/min & $88.49 \pm 19.25$ & $92.87 \pm 26.25$ & 1.262 & 0.209 \\
\hline Respiratory frequency, breaths/min & $24.26 \pm 7.13$ & $26.31 \pm 8.84$ & 1.358 & 0.176 \\
\hline Body temperature, ${ }^{\circ} \mathrm{C}$ & $36.29 \pm 0.914$ & $36.34 \pm 0.71$ & 0.429 & 0.668 \\
\hline White blood cells, per $10^{9} / 1$ & $12.30 \pm 4.39$ & $13.65 \pm 5.22$ & 1.891 & 0.060 \\
\hline Hemoglobin, g/dl & $14.07 \pm 4.15$ & $14.45 \pm 5.86$ & 0.499 & 0.619 \\
\hline Platelet, per $10^{9} / 1$ & $243.77 \pm 89.32$ & $251.04 \pm 101.74$ & 0.515 & 0.607 \\
\hline Alanine aminotransferase, U/1 & $44.74 \pm 25.89$ & $52.04 \pm 30.03$ & 1.760 & 0.080 \\
\hline Total bilirubin, $\mu \mathrm{mol} / 1$ & $21.14 \pm 12.56$ & $24.09 \pm 12.52$ & 1.613 & 0.108 \\
\hline Albumin, g/l & $37.97 \pm 9.82$ & $36.26 \pm 10.70$ & 0.158 & 0.248 \\
\hline Serum amylase, U/1 & $90.65 \pm 42.66$ & $97.52 \pm 46.22$ & 1.052 & 0.294 \\
\hline Serum lipase, U/1 & $101.14 \pm 38.27$ & $112.63 \pm 58.14$ & 1.659 & 0.099 \\
\hline Urea nitrogen, mmol/1 & $7.69 \pm 3.79$ & $8.40 \pm 4.64$ & 1.129 & 0.260 \\
\hline Creatinine, $\mu \mathrm{mol} / 1$ & $92.83 \pm 44.39$ & $106.38 \pm 56.22$ & 1.873 & 0.063 \\
\hline Serum CK-MB isoenzyme, U/l & $20.26 \pm 13.15$ & $23.19 \pm 12.98$ & 1.361 & 0.175 \\
\hline Serum troponin I, ng/ml & $0.23 \pm 0.14$ & $0.27 \pm 0.19$ & 1.688 & 0.093 \\
\hline Blood glucose, mmol/1 & $9.70 \pm 5.41$ & $10.79 \pm 5.84$ & 1.319 & 0.189 \\
\hline Serum potassium, mmol/l & $4.24 \pm 1.39$ & $3.94 \pm 2.05$ & 1.138 & 0.257 \\
\hline Serum sodium, mmol/1 & $140.35 \pm 41.95$ & $137.96 \pm 40.86$ & 0.397 & 0.692 \\
\hline $\mathrm{pH}$ & $7.36 \pm 0.25$ & $7.40 \pm 0.30$ & 0.978 & 0.329 \\
\hline $\mathrm{PaO}_{2}, \mathrm{mmHg}$ & $94.87 \pm 33.03$ & $97.70 \pm 40.66$ & 0.515 & 0.607 \\
\hline $\mathrm{PaCO}_{2}, \mathrm{mmHg}$ & $32.86 \pm 10.24$ & $30.41 \pm 7.8$ & 1.804 & 0.073 \\
\hline $\mathrm{HCO}_{3}^{-}, \mathrm{mmol} / 1$ & $21.31 \pm 4.27$ & $20.31 \pm 3.51$ & 1.785 & 0.076 \\
\hline Base excess, mmol/1 & $-2.82 \pm 1.35$ & $-3.21 \pm 1.89$ & 1.677 & 0.095 \\
\hline Arterial blood lactate, $\mathrm{mmol} / \mathrm{l}$ & $1.50 \pm 0.97$ & $2.90 \pm 1.21$ & 8.925 & $<0.001^{\mathrm{a}}$ \\
\hline $\mathrm{C}_{0}, \mu \mathrm{g} / \mathrm{ml}$ & $20.28 \pm 19.95$ & $48.67 \pm 17.27$ & 11.350 & $<0.001^{\mathrm{a}}$ \\
\hline APACHE II & $8.74 \pm 6.16$ & $11.98 \pm 7.02$ & 3.326 & $<0.001^{\mathrm{a}}$ \\
\hline
\end{tabular}

${ }^{a} \mathrm{P}<0.05$ between the survival group and mortality group. $\mathrm{CK}$, creatine kinase; $\mathrm{C}_{0}$, initial concentration of paraquat in urine; APACHE II, Acute Physiology and Chronic Health Evaluation II.

PQ concentration was obtained and the PQ concentration in the urine of patients was quantitatively detected.

A total of $100 \mathrm{~g}$ sodium dithionite was added to sodium hydroxide solution $(2 \mathrm{ml} ; 2 \mathrm{M})$ and remained stable for approximately $2 \mathrm{~h}$. Urine samples were collected from patients in colorless tubes and maintained at $-20^{\circ} \mathrm{C}$ for $10 \mathrm{~min}$, followed by centrifugation at $9660 \mathrm{x}$ g for $5 \mathrm{~min}$ at room temperature. A total of $200 \mu \mathrm{l}$ alkaline dithionite solution was added to $2 \mathrm{ml}$ of the pretreated urine. Following gentle mixing, $50 \mu \mathrm{l}$ supernatant was collected to measure the absorbance. Absorbance was measured at $395 \mathrm{~nm}$ and a working curve of concentration-absorbance was prepared. Linear regression curve: $y=0.0895 x+0.0151$, with a correlation coefficient of $r=0.9999$ when the urine PQ concentration was 0.01 to $100 \mu \mathrm{g} / \mathrm{ml}$. Recovery rate ranged from 92.5 to $104.0 \%$, with relative standard deviation (RSD) ranging from 2.7 to $4.5 \%$, and the limit of detection (LOD) was $0.01 \mu \mathrm{g} / \mathrm{ml}$. According to the test sample and above formula, the urine PQ concentration was calculated.

Statistical analysis. SPSS 18.0 software (SPSS, Inc., Chicago, IL, USA) was used for statistical analysis. Measurement data were expressed as the mean \pm standard deviation, Student's $\mathrm{t}$-test was applied to detect inter-group comparisons, and $\chi^{2}$ test was performed to detect enumeration data. Valuable detection indices were screened out by logistic regression analysis and ROC curve analysis and Kaplan-Meier curve was applied to evaluate their diagnostic efficiency. $\mathrm{P}<0.05$ was considered to indicate a statistically significant difference. 
Table II. Logistic regression analysis for dose of poison, initial concentration of paraquat in urine, arterial blood lactate and APACHE II scores.

$95 \% \mathrm{CI}$

\begin{tabular}{lcrrrrrr}
\cline { 5 - 7 } Variable & B & S.E & Wals & P-value & Exp (B) & LL & UL \\
\hline Dose of poison, ml & 0.078 & 0.017 & 20.981 & $<0.001$ & 1.081 & 1.046 & 1.118 \\
Initial concentration of paraquat in urine $\left(\mathrm{C}_{0}\right), \mu \mathrm{g} / \mathrm{ml}$ & 0.073 & 0.018 & 16.023 & $<0.001$ & 1.076 & 1.038 & 1.115 \\
Arterial blood lactate, $\mathrm{mmol} / \mathrm{l}$ & 0.948 & 0.290 & 10.682 & 0.001 & 2.580 & 1.462 & 4.556 \\
APACHE II scores & 0.054 & 0.044 & 1.472 & 0.225 & 1.055 & 0.967 & 1.151 \\
\hline
\end{tabular}

APACHE II, Acute Physiology And Chronic Health Evaluation II; B, regression coefficient; S.E, standard error; Wals, Wald $\chi^{2}$ value; Exp (B), adjusted odd ratio; 95\% CI, 95\% confidence interval; LL, lower limit; UL, upper limit.

Table III. ROC curve analysis for the initial concentration of paraquat in urine, dose of poison, arterial blood lactate and APACHE II scores in predicting fatality rate within 28 days.

\begin{tabular}{lccrrr}
\hline Variable & $\begin{array}{c}\text { Area under } \\
\text { ROC curve }\end{array}$ & $95 \%$ CI & $\begin{array}{c}\text { Cutoff } \\
\text { value }\end{array}$ & $\begin{array}{c}\text { Sensitivity } \\
(\%)\end{array}$ & $\begin{array}{c}\text { Specificity } \\
(\%)\end{array}$ \\
\hline Initial concentration of paraquat in urine & 0.921 & $0.880-0.961$ & 32.20 & 91.20 & 80.00 \\
Dose of poison & 0.887 & $0.842-0.931$ & 46.50 & 73.70 & 0.712 \\
Arterial blood lactate & 0.808 & $0.747-0.869$ & 2.45 & 69.28 & 75.01 \\
APACHE II & 0.648 & $0.570-0.726$ & 6.50 & 78.11 & 47.52 \\
\hline
\end{tabular}

ROC, receiver operator characteristic; 95\% CI, 95\% confidence interval; APACHE II, Acute Physiology and Chronic Health Evaluation II.

\section{Results}

Comparisons of baseline characteristics. The 194 patients were all poisoned with oral doses of PQ. Within the 28-day follow-up period after poisoning, 114 patients succumbed to poisoining and 80 patients survived, with a mortality rate of $58.8 \%$. Dose of poison, arterial blood lactate, initial urine PQ concentration and APACHE II score were all significantly higher in the mortality group compared with the survival group (all $\mathrm{P}<0.05)$. Additionally, no significant difference in the comparisons of other indices, including but not limited to mean arterial pressure, heart rate, respiratory frequency and body temperature, were observed between the two groups (all P>0.05; Table I).

Logistic regression analysis. Logistic regression analysis was performed on the independent variables that had statistical differences in single factor analysis, suggesting that only dose of poison (OR, 1.081; 95\% CI, 1.046-1.118; $\mathrm{P}<0.001)$, initial urine $\mathrm{PQ}$ concentration $\left(\mathrm{C}_{0}\right)(\mathrm{OR}, 1.076 ; 95 \% \mathrm{CI}$, 1.038-1.115; $\mathrm{P}<0.001)$ and arterial blood lactate $(\mathrm{OR}, 2.580$; $95 \% \mathrm{CI}, 1.462-4.556 ; \mathrm{P}=0.001)$ were associated with the risk of mortality from PQ poisoning (Table II).

Receiver operating characteristic curve analysis. Results of ROC curve analysis of dose of poison, initial urine PQ concentration and arterial blood lactate in predicting the mortality rate of patients within 28 days of poisoning are presented in Table III and Fig. 1. The area under the curve (AUC) of initial urine $P Q$ concentration $\left(\mathrm{C}_{0}\right)$ was the largest at 0.921 , which has a relatively more accurate discrimination for patient prognosis. The AUC of dose of poison and arterial blood lactate were 0.887 and 0.808 , respectively, and their discrimination for the prognosis of acute PQ poisoning was only second to initial urine PQ concentration $\left(\mathrm{C}_{0}\right)$. The AUC of APACHE II score was 0.648 , suggesting it is relatively poor at predicting the prognosis of acute $\mathrm{PQ}$ poisoning.

Among the 80 patients in the survival group and 114 in the mortality group, 20 patients died within 7 days of poisoning, including 11 from sudden cardiac arrest and 9 from multiple organ failure. The remaining 94 patients died within 7-28 days of poisoning, including 81 from refractory hypoxemic respiratory failure (minimum value of arterial partial pressure of oxygen, $27-46 \mathrm{mmHg}$; mean value, $38 \pm 16 \mathrm{mmHg}$ ) and 13 from multiple organ failure. Initial urine PQ concentration of patients in the early and delayed mortality groups was significantly higher than in the survival group (all $\mathrm{P}<0.01$ ), and initial urine $\mathrm{PQ}$ concentration in early mortality group was significantly higher compared with the delayed mortality group $(86.73 \pm 16.62$ vs. $40.57 \pm 12.36$; $\mathrm{t}=3.808 ; \mathrm{P}=0.001$ ) (Fig. 2). The AUC of the initial urine PQ concentration of patients in predicting early mortality after poisoning was 0.890 (95\% CI, 0.837-0.944) with a cutoff value of $49.3 \mu \mathrm{g} / \mathrm{ml}$, sensitivity and specificity values of 100.0 and $69.0 \%$, respectively, and a Youden index score of 0.690 (Fig. 3). The AUC of the initial urine PQ concentration of patients in predicting delayed mortality was 0.764 (95\% CI, $0.693-0.834)$ with a cutoff value of $37.5 \mu \mathrm{g} / \mathrm{ml}$, sensitivity 


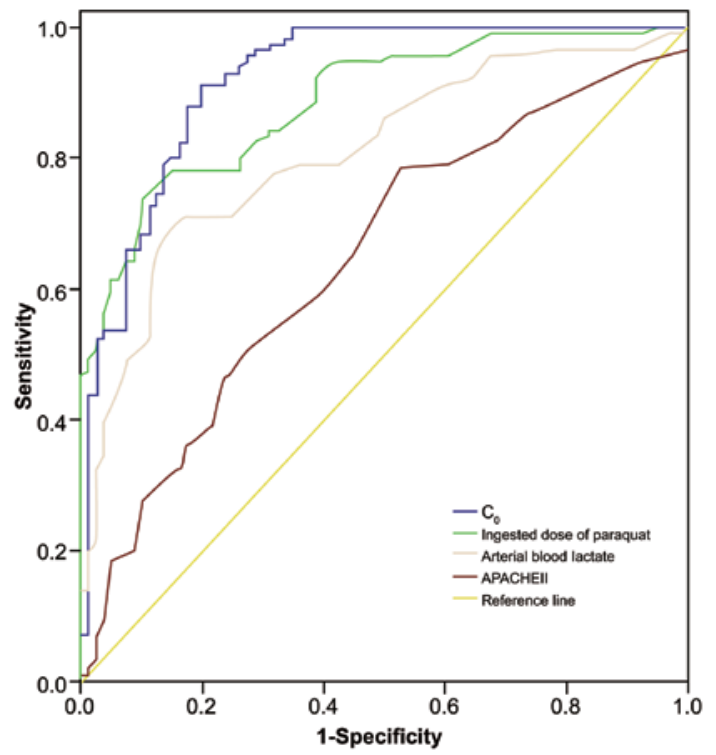

Figure 1. Receiver operating characteristic curve analysis of $\mathrm{C}_{0}$, dose of poison, arterial blood lactate and APACHE II in predicting the mortality rate of patients with paraquat poisoning within 28 days of poisoning. $\mathrm{C}_{0}$, initial urine paraquat concentration; APACHE II, Acute Physiology and Chronic Health Evaluation II.

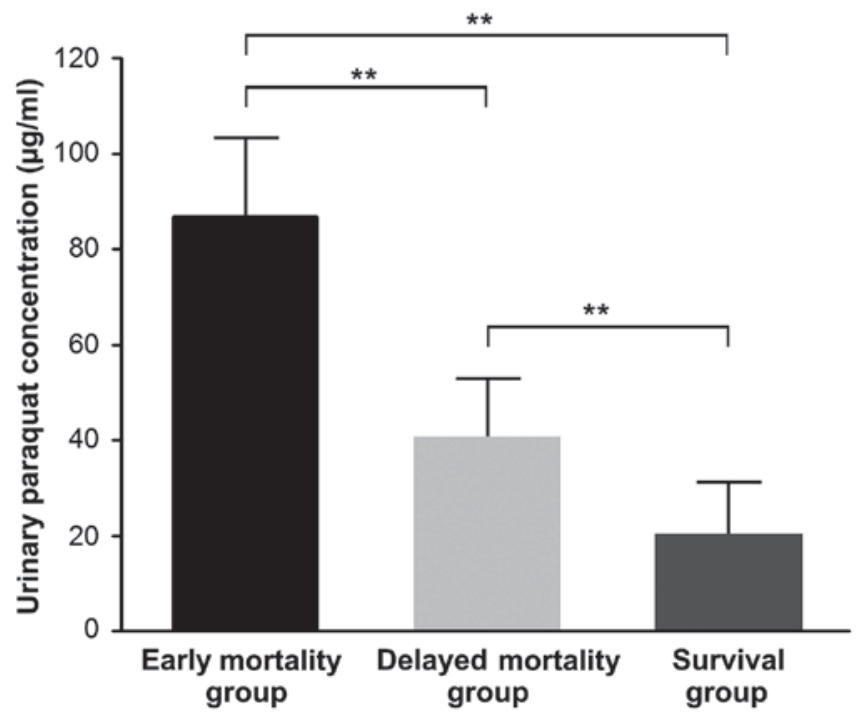

Figure 2. Comparisons of initial urine paraquat concentration between the three groups. ${ }^{* *} \mathrm{P}<0.01$.

and specificity values of 89.4 and $64.0 \%$, respectively, and a Youden index score of 0.534 (Fig. 4). These findings indicate that the efficacy of the initial urine PQ concentration in predicting early mortality was higher when compared with delayed mortality.

Prognostic value of urine paraquart concentration. Patients were divided into two groups according to the initial urine PQ concentration $\left(\mathrm{C}_{0}, 32.2 \mu \mathrm{g} / \mathrm{ml}\right)$. During the observation period (28-day follow-up after poisoning), the survival rate of patients with $\mathrm{C}_{0} \leq 32.2 \mu \mathrm{g} / \mathrm{ml}(42 / 71 ; 59.2 \%)$ was significantly higher when compared with those with $\mathrm{C}_{0}>32.2 \mu \mathrm{g} / \mathrm{ml}(38 / 123$; $30.9 \%$; Fig. 5), suggesting that $\mathrm{C}_{0}$ was an important index for

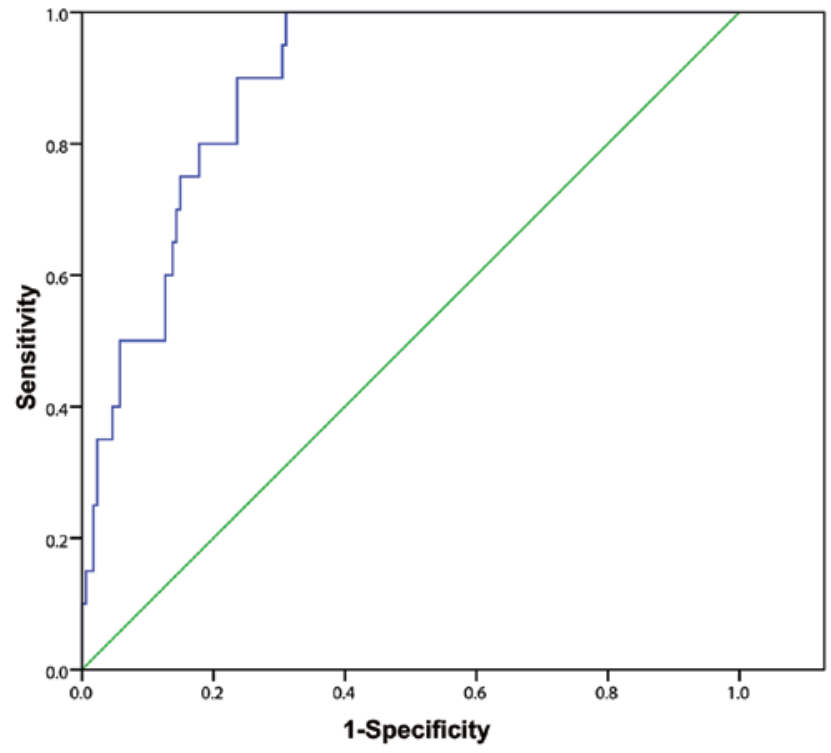

Figure 3. Receiver operating characteristic curve analysis of initial urine paraquat concentration in predicting early mortality rate in patients with paraquat poisoning.

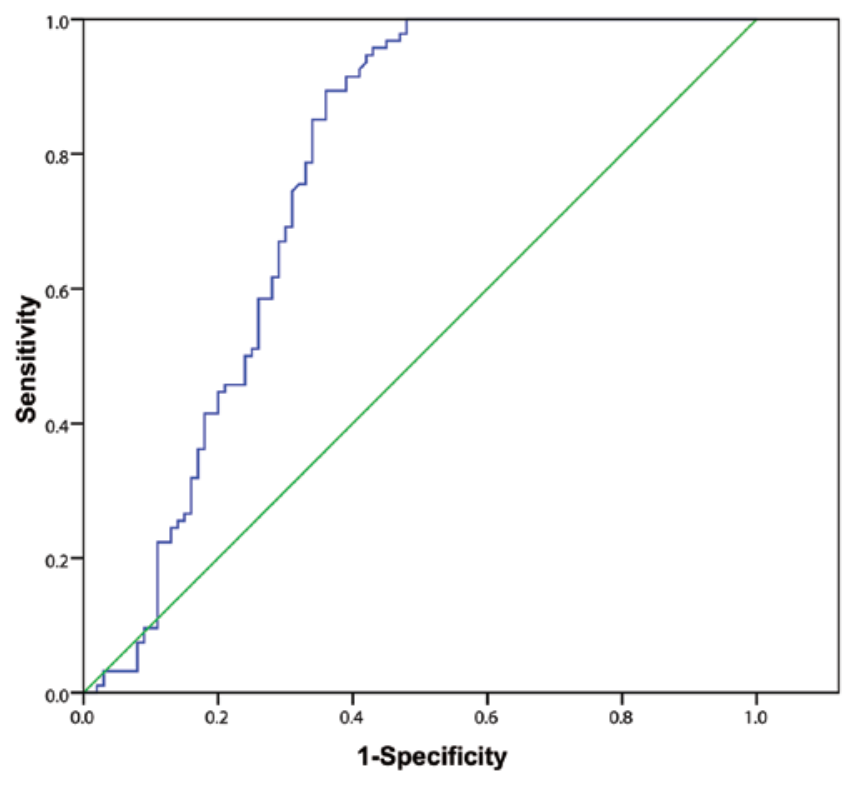

Figure 4. Receiver operating characteristic curves of initial urine paraquat concentration in predicting delayed mortality rate in patients with paraquat poisoning.

predicting mortality rate in patients within 28 days (OR: 14.33 , 95\% CI, 1.728-5.838; $\left.\chi^{2}=14.33 ; \mathrm{P}<0.001\right)$.

Rebound rate of urine paraquart concentration after hemoperfusion. Selected patients received HP 2-5 times (3.7 \pm 2.2$)$ within $24 \mathrm{~h}$ after poisoning, among which 11 received HP 5 times, 123 received HP 4 times, 46 received HP 3 times and 14 received HP 2 times. Urine PQ concentration levels in patients who received HP 4 times within $24 \mathrm{~h}$ after poisoning was dynamically monitored. We found that the average urine PQ concentration of patients in the mortality and survival groups dropped to $1 \mu \mathrm{g} / \mathrm{ml}$, but both rebounded the next day to different degrees. On the day after poisoning, urine PQ 
Table IV. Changes in urine paraquat concentration before and after blood perfusion in patients with acute paraquat poisoning.

\begin{tabular}{lccccc}
\hline Urine paraquat concentration $(\mu \mathrm{g} / \mathrm{ml})$ & $\mathrm{C}_{0}$ & $\mathrm{C}_{1}$ & $\mathrm{C}_{4}$ & $\mathrm{C}_{\text {sec }}$ & Rebound rate $(\%)$ \\
\hline Mortality group $(\mathrm{n}=72)$ & $48.67 \pm 17.27^{\mathrm{a}}$ & $16.29 \pm 6.97^{\mathrm{a}, \mathrm{b}}$ & $0.66 \pm 0.32^{\mathrm{a}, \mathrm{b}}$ & $0.94 \pm 0.38^{\mathrm{a}, \mathrm{b}}$ & $39.80 \pm 28.56^{\mathrm{a}}$ \\
Survival group $(\mathrm{n}=51)$ & $20.28 \pm 19.95$ & $12.44 \pm 6.06^{\mathrm{b}}$ & $0.25 \pm 0.13^{\mathrm{b}}$ & $0.28 \pm 0.16^{\mathrm{b}}$ & $13.71 \pm 18.97$ \\
\hline
\end{tabular}

Rebound rate was calculated as: Rebound rate $\left.=\left(\mathrm{C}_{\mathrm{sec}}-\mathrm{C}_{4}\right) / \mathrm{C}_{4}\right)$. ${ }^{\mathrm{P}}<0.01 \mathrm{vs}$. the survival group; ${ }^{\text {b }}<0.01 \mathrm{vs}$. $\mathrm{C}_{0}$. $\mathrm{C}_{0}$, initial urine paraquat concentration; $\mathrm{C}_{1}$, urine paraquat concentration after a single hemoperfusion; $\mathrm{C}_{4}$, urine paraquat concentration after four rounds of hemoperfusion; $\mathrm{C}_{\mathrm{sec}}$, urine PQ concentration the following day after hemoperfusion.

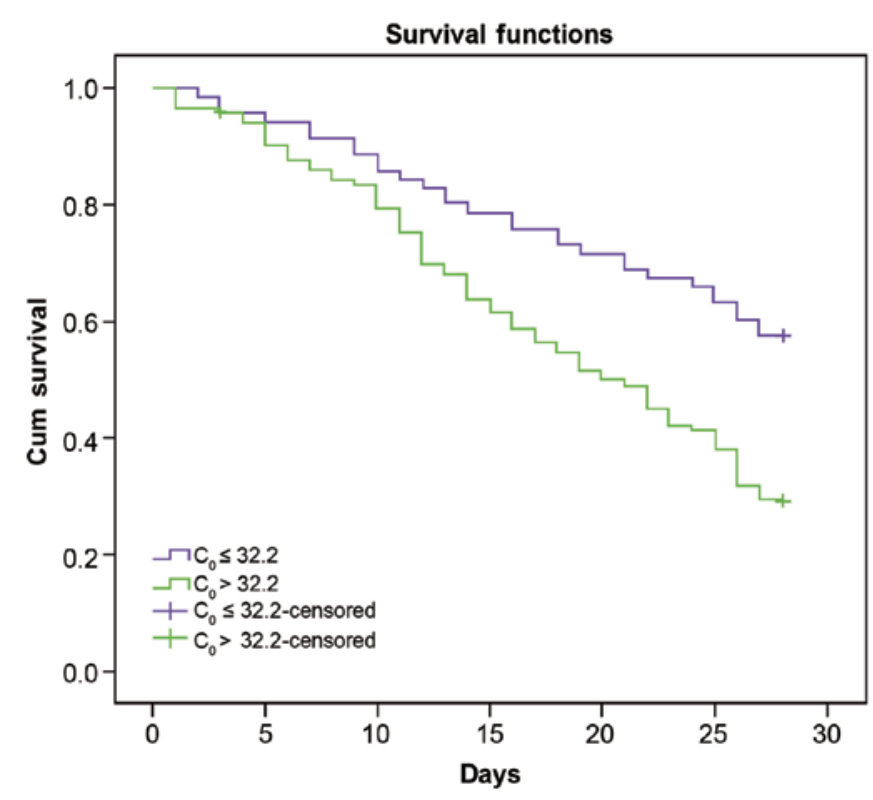

Figure 5. Influence of $\mathrm{C}_{0}$ on the survival rate of patients with paraquat poisoning. $\mathrm{C}_{0}$, initial urine paraquat concentration; $\mathrm{Cum}$, cumulative.

concentration in the mortality group was significantly higher compared with the survival group $(t=2.232 ; \mathrm{P}=0.036)$. The rebound rate in the mortality group was also markedly higher compared with the survival group $(t=2.254 ; \mathrm{P}=0.022$; Table IV).

In the 123 patients that received HP 4 times within $24 \mathrm{~h}$ of poisoning, the area under the ROC curve of urine PQ concentration on the day after $\mathrm{HP}\left(\mathrm{C}_{\mathrm{sec}}\right)$ in predicting the mortality rate within 28 days was 0.919 (95\% CI, 0.878-0.960; $\mathrm{P}<0.001)$. The area under the ROC curve of the rebound rate of urine PQ concentration on the next day in predicting mortality rate within 28 days was 0.805 (95\% CI, 0.742-0.867; $\mathrm{P}<0.001$; Fig. 6).

\section{Discussion}

This study investigated the efficacy of urine PQ concentration, dose of poison, arterial blood lactate and APACHE II scoring in predicting the prognosis of patients with acute PQ poisoning. By comparing indices between the survival and mortality groups, we found that initial urine PQ concentration, dose of poison, arterial blood lactate and the APACHE II score of patients in the mortality group were all significantly higher compared with the survival

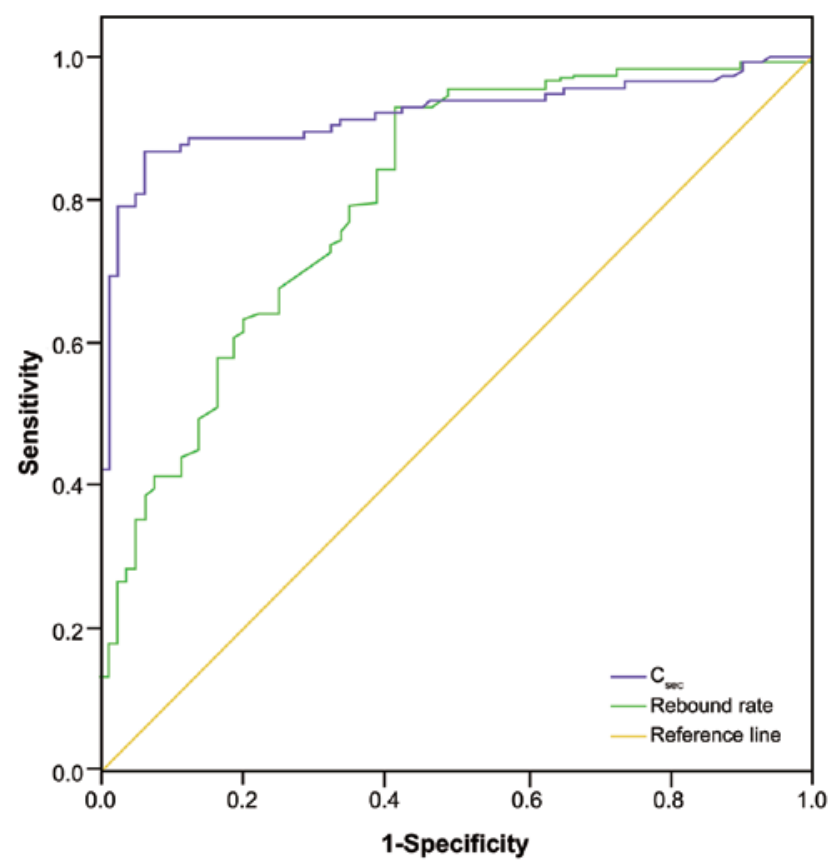

Figure 6. Receiver operating characteristic curve analysis of rebound rate and $\mathrm{C}_{\mathrm{sec}}$ in predicting the mortality rate of patients with paraquat poisoning within 28 days of poisoning. Rebound rate was calculated as: Rebound rate $\left.=\left(\mathrm{C}_{\mathrm{sec}}-\mathrm{C}_{4}\right) / \mathrm{C}_{4}\right) . \mathrm{C}_{\mathrm{sec}}$, urine paraquat concentration on the day after poisoning; $\mathrm{C}_{4}$, urine paraquat concentration after four rounds of hemoperfusion.

group, suggesting that all four indices were able to predict the prognosis of patients with acute PQ poisoning to some extent, compared with other indices, such as arterial pressure, heart rate and serum potassium. Consistent with our results, Ruan et al (7) reported that the PQ concentration in urine served as an invaluable predictive index for the prognosis of patients in acute PQ poisoning and, in another study, urine PQ concentrations reflected the severity of acute PQ poisoning (18). PQ can cause acute tubular necrosis and therefore lead to renal failure at high doses (19). However, if patients ingest $<40 \mathrm{mg} / \mathrm{kg} \mathrm{PQ}$, renal damage is reversible and the mortality rate is lower compared with patients who have ingested higher doses of PQ (20). Consequently, dose of PQ is a stable index for predicting the prognosis of patients with acute PQ poisoning. APACHE II is a severity-of-disease classification system applied within $24 \mathrm{~h}$ of admission of a patient to the Intensive Care Unit (12). It has also been used as a proxy indicator to reflect the intermediate effect of PQ poisoning dose and the severity of PQ intoxication 
responses (21). An integer score from 0 to 71 is computed based on several measurements. The higher scores correspond to more severe disease and a higher risk of mortality. Consistent with our results, Chang et al (22) revealed that APACHE II scores $>9$ had a sensitivity of $64 \%$ and a specificity of $88 \%$ in predicting 30-day mortality in acute PQ-poisoned patients. PQ produces reactive oxygen species, which have a leading role in causing multiple organ failure and the elevated lactate concentration in the serum is a manifestation of the underlying organ dysfunction (13). Our results further support the predictive power of arterial blood lactate in evaluating the prognosis of patients with acute PQ poisoning. Consistent with our study outcomes, Lee et al (9) also revealed that arterial lactate measurement provides useful information for patients with acute PQ poisoning and may potentially lead to improved decision making.

In the present study, multiple factor logistic regression analysis revealed that only dose of poison, initial urine PQ concentration $\left(\mathrm{C}_{0}\right)$ and arterial blood lactate were associated with the risk of mortality from PQ poisoning, suggesting that, compared with APACHE II scoring, dose of poison, initial urine PQ concentration and arterial blood lactate were more sensitive in predicting the mortality risk of PQ poisoning. However, in direct contrast with our results, a study by Huang et al (21), showed that an APACHE II score of $>13$, calculated $24 \mathrm{~h}$ after admission, predicted in-hospital mortality with relatively high sensitivity (67\%) and specificity (94\%) and concluded that the APACHE II system yielded superior discriminative power than plasma PQ concentration or estimated PQ ingestion dosage. Limitations of the APACHE II system, including reduced applicability in patients with more severe disease, may contribute to this difference (21). and APACHE II scoring does not include parameters reflecting liver damage, which is a major complication in PQ poisoning (15). Therefore, evaluation of APACHE II score is complex and not advisable for typical hospital inpatients (20).

ROC curve analysis ranked the accuracy of the three indices as follows: Initial urine PQ concentration with the best accuracy, dose of poison, and arterial blood lactate. Though arterial blood lactate is a useful index in predicting the prognosis of acute PQ poisoning patients, determination of the circulating levels of lactate is more practical and technically simpler, is frequently used, and is a clinically available technique with a rapid turnaround (23). Initial urine PQ concentrations, and a comparison of urine PQ concentrations associated with patient survival, in the early and delayed mortality groups suggested that the initial urine PQ concentration of patients in the early and delayed mortality groups was significantly higher compared with the survival group. Notably, initial urine PQ concentration in the early mortality group was also significantly higher compared with the delayed mortality group. ROC curve analysis suggested that the efficacy of initial urine PQ concentration in predicting early mortality was higher than its efficiency in predicting delayed mortality. The survival rate of patients with $\mathrm{C}_{0} \leq 32.2 \mu \mathrm{g} / \mathrm{ml}$ was significantly higher when compared with patients exhibiting $\mathrm{C}_{0}>32.2 \mu \mathrm{g} / \mathrm{ml}$, suggesting that $\mathrm{C}_{0}$ is an important index for predicting mortality rate within the 28-day period. In addition, the rebound rate of PQ concentration in the mortality group was also markedly higher when compared with the survival group, suggesting that the rebound rate may be an important risk factor for fatality from acute $\mathrm{PQ}$ poisoning and may have a predictive role in the prognosis of patients with acute PQ poisoning.

In conclusion, the initial urine PQ concentration may be the optimal index for predicting the prognosis of patients with acute PQ poisoning. Additionally, dose of poison, arterial blood lactate, $\mathrm{C}_{\mathrm{sec}}$ and the rebound rate also have has some referential significance. In view of the relatively small sample size, the results of this study will need to be confirmed in future studies that involve larger sample sizes.

\section{References}

1. Sun IO and Lee KY: Cyclophosphamide dose: How much is needed to win the war against paraquat poisoning? Korean J Intern Med 28: 410-412, 2013.

2. Liu XW, Ma T, Qu B, Ji Y and Liu Z: Prognostic value of initial arterial lactate level and lactate metabolic clearance rate in patients with acute paraquat poisoning. Am J Emerg Med 31: 1230-1235, 2013.

3. Wunnapuk K, Mohammed F, Gawarammana I, Liu X, Verbeeck RK, Buckley NA, Roberts MS and Musuamba FT: Prediction of paraquat exposure and toxicity in clinically ill poisoned patients: A model based approach. Br J Clin Pharmacol 78: 855-866, 2014.

4. Dinis-Oliveira RJ, Duarte JA, Sánchez-Navarro A, Remião F, Bastos ML and Carvalho F: Paraquat poisonings: Mechanisms of lung toxicity, clinical features, and treatment. Crit Rev Toxicol 38: 13-71, 2008.

5. Wu B, Song B, Yang H, Huang B, Chi B, Guo Y and Liu H: Central nervous system damage due to acute paraquat poisoning: An experimental study with rat model. Neurotoxicology 35: 62-70, 2013.

6. Liu Z, Zhao H, Liu W, Li T, Wang Y and Zhao M: NLRP3 inflammasome activation is essential for paraquat-induced acute lung injury. Inflammation 38: 433-444, 2015.

7. Ruan XL, Qiu JJ, Wu C, Huang T, Meng RB and Lai YQ: Magnetic single-walled carbon nanotubes-dispersive solid-phase extraction method combined with liquid chromatography-tandem mass spectrometry for the determination of paraquat in urine. J Chromatogr B Analyt Technol Biomed Life Sci 965: 85-90, 2014.

8. Yeo CD, Kim JW, Kim YO, Yoon SA, Kim KH and Kim YS: The role of pentraxin-3 as a prognostic biomarker in paraquat poisoning. Toxicol Lett 212: 157-160, 2012.

9. Lee Y, Lee JH, Seong AJ, Hong CK, Lee HJ, Shin DH and Hwang SY: Arterial lactate as a predictor of mortality in emergency department patients with paraquat intoxication. Clin Toxicol (Phila) 50: 52-56, 2012.

10. Seok S, Kim YH, Gil HW, Song HY and Hong SY: The time between paraquat ingestion and a negative dithionite urine test in an independent risk factor for death and organ failure in acute paraquat intoxication. J Korean Med Sci 27: 993-998, 2012.

11. Gil HW, Kang MS, Yang JO, Lee EY and Hong SY: Association between plasma paraquat level and outcome of paraquat poisoning in 375 paraquat poisoning patients. Clin Toxicol (Phila) 46: 515-518, 2008.

12. Hassanian-Moghaddam H, Amiri H, Zamani N, Rahimi M, Shadnia S and Taherkhani M: QT dispersion and prognostication of the outcome in acute cardiotoxicities: A comparison with SAPS II and APACHE II scoring systems. Cardiovasc Toxicol 14: 129-133, 2014.

13. Mikkelsen ME, Miltiades AN, Gaieski DF, Goyal M, Fuchs BD, Shah CV, Bellamy SL and Christie JD: Serum lactate is associated with mortality in severe sepsis independent of organ failure and shock. Crit Care Med 37: 1670-1677, 2009.

14. Weil MH and Tang W: Clinical correlates of arterial lactate levels in STEMI patients. Crit Care 15: 113, 2011.

15. Yang CJ, Lin JL, Lin-Tan DT, Weng CH, Hsu CW, Lee SY, Lee SH, Chang CM, Lin WR and Yen TH: Spectrum of toxic hepatitis following intentional paraquat ingestion: Analysis of 187 cases. Liver Int 32: 1400-1406, 2012. 
16. M PN: World Medical Association publishes the Revised Declaration of Helsinki. Natl Med J India 27: 56, 2014.

17. Berry DJ and Grove J: The determination of paraquat (I,I'-dimethyl-4,4'-bipyridylium cation) in urine. Clin Chim Acta 34: 5-11, 1971

18. Liu Z, Liu D, Zhang X, Wang $\mathrm{H}$ and Hao F: Prognosis value of urine paraquat semi-quantitative in the patients with acute paraquat poisoning. Chinese Journal of Industrial Hygiene and Occupational Diseases 32: 378-380, 2014 (In Chinese).

19. Singh AP, Junemann A, Muthuraman A, Jaggi AS, Singh N, Grover K and Dhawan R: Animal models of acute renal failure. Pharmacol Rep 64: 31-44, 2012.

20. Weng CH, Hu CC, Lin JL, Lin-Tan DT, Huang WH, Hsu CW and Yen TH: Sequential organ failure assessment score can predict mortality in patients with paraquat intoxication. PLoS One 7: e51743, 2012.
21. Huang NC, Hung YM, Lin SL, Wann SR, Hsu CW, Ger LP, Hung SY, Chung HM and Yeh JH: Further evidence of the usefulness of acute physiology and chronic health evaluation II scoring system in acute paraquat poisoning. Clin Toxicol (Phila) 44: 99-102, 2006.

22. Chang MW, Chang SS, Lee CC, Sheu BF and Young YR: Hypokalemia and hypothermia are associated with 30-day mortality in patients with acute paraquat poisoning. Am J Med Sci 335: 451-456, 2008.

23. Howell MD, Donnino M, Clardy P, Talmor D and Shapiro NI: Occult hypoperfusion and mortality in patients with suspected infection. Intensive Care Med 33: 1892-1899, 2007. 